\title{
Modeling Analysis and Structural Design of Human Lower Limb Rehabilitation Robot
}

\author{
Zhiming Wang, Lizhen Cui ${ }^{a}$, Zhenglong Cai, Changfu Pang \\ School of Mechatronic Engineering and Automation, Shanghai University, Shanghai 200072, China
}

\begin{abstract}
With the rapid development of science and technology, robots are widely used in rehabilitation training. According to the physiological structure of human lower limbs and gait characteristics of walking, a lower limb rehabilitation robot is designed in this paper. We design the structure in a form of exoskeleton with three degrees of freedom in which kinematics analysis is carried out by the $\mathrm{D}-\mathrm{H}$ coordinate transformation method. And then we obtain the relationship between the end effector and the angle of each joint. In addition, the relationship between end effector speed and joint speed is obtained through Jacobian matrix and Lagrange equilibrium method is used for dynamic analysis. The joint torque is calculated through the joint speed and three dimensional modeling of lower limb rehabilitation robot was reconstructed by Pro-e. Finally, the driving mode is selected and calculated.
\end{abstract}

\section{Introduction}

As we all know, the lower limbs of the human body are divided into thigh bone, leg bone and foot bone. Also the lower extremities have three joints including hip, knee and ankle. They work together to form the movement of human lower extremities. There are three degrees of freedom in the motion of hip joint including flexion or extension, abduction or adduction and external rotation or internal rotation. Also there are two degrees of freedom during the motion of knee, including flexion or extension and external rotation or internal rotation. The motion of the ankle is divided into two degrees of freedom such as dorsiflexion or metatarsal flexion and varus or valgus. Normally, in the course of human walking, the balance of body is mainly done by abduction of joints. External rotation mainly changes the direction of movement [1]. Step movements are mainly done by flexion and extension at joints of lower extremities. Therefore, on the basis of routine rehabilitation training, the degree of anterior flexion or extension of hip joint is reserved only.

\section{Modeling analysis}

\subsection{Kinematic modeling and calculation}

\subsubsection{Position analysis}

The exoskeleton mechanical leg is simplified into a linkage mechanism, and Cartesian coordinate system $\{i\}$ is established at each link. The relationship between each link is represented by homogeneous transformation matrix by $\mathrm{D}-\mathrm{H}$ method [2]. Then we determine the pose of the robot's end and the pose of the robot's end effector. The coordinate system of exoskeleton robot legs for lower limb rehabilitation robot is shown in Figure 2.1.

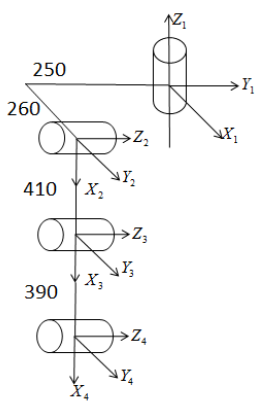

Figure 2.1. D-H coordinate system

There is a rotational degree of freedom at the hip, knee and ankle joints. The parameters of the connecting rods are shown in Table 2.1.

Table 2.1. D-H parameters

\begin{tabular}{ccccc}
\hline Link i & $\theta_{i}$ & $\alpha_{i-1}$ & $d_{i}$ & $a_{i-1}$ \\
\hline 1 & $\theta_{1}$ & 0 & 25 & 0 \\
2 & $\theta_{2}$ & $90^{\circ}$ & 26 & 0 \\
3 & $\theta_{3}$ & 0 & 0 & 410 \\
4 & $\theta_{4}$ & 0 & 0 & 390 \\
\hline
\end{tabular}

The forward kinematics solution is to calculate position and attitude of the end effector coordinate system in the base coordinate system with known joint displacement or angle. The transformation matrix of connecting rod $\mathrm{i}$ relative to adjacent link $\mathrm{i}-1$ is $:{ }_{i}^{i-1} T=$ 
$\left[\begin{array}{cccc}\cos \theta_{i} & -\sin \theta_{i} & 0 & a_{i-1} \\ \sin \theta_{i} \cos \alpha_{i-1} & \cos \theta_{i} \cos \alpha_{i-1} & -\sin \alpha_{i-1} & -d_{i} \sin \alpha_{i-1} \\ \sin \theta_{i} \sin \alpha_{i-1} & \cos \theta_{i} \sin \alpha_{i-1} & \cos \alpha_{i-1} & d_{i} \cos \alpha_{i-1} \\ 0 & 0 & 0 & 1\end{array}\right]$

The transformation matrices between lower limb linkage coordinate systems are as follows:

${ }_{1}^{0} T=\left[\begin{array}{cccc}\cos \theta_{1} & -\sin \theta_{1} & 0 & 0 \\ \sin \theta_{1} & \cos \theta_{1} & 0 & 0 \\ 0 & 0 & 1 & 250 \\ 0 & 0 & 0 & 1\end{array}\right]$
${ }_{2}^{1} T=\left[\begin{array}{cccc}\cos \theta_{2} & -\sin \theta_{2} & 0 & 0 \\ 0 & 0 & -1 & -260 \\ \sin \theta_{2} & \cos \theta_{2} & 0 & 0 \\ 0 & 0 & 0 & 1\end{array}\right]$
${ }_{3}^{2} T=\left[\begin{array}{cccc}\cos \theta_{3} & -\sin \theta_{3} & 0 & 410 \\ \sin \theta_{3} & \cos \theta_{3} & 0 & 0 \\ 0 & 0 & 1 & 0 \\ 0 & 0 & 0 & 1\end{array}\right]$
${ }_{4}^{3} T=\left[\begin{array}{cccc}\cos \theta_{4} & -\sin \theta_{4} & 0 & 390 \\ \sin \theta_{4} & \cos \theta_{4} & 0 & 0 \\ 0 & 0 & 1 & 0 \\ 0 & 0 & 0 & 1\end{array}\right]$

In the coordinate system $\mathrm{J}$, the transformation matrix of the coordinate system $\mathrm{K}$ can be obtained by multiplication: ${ }_{k}^{j} T=\prod_{i=j i+1}^{k-1} T$. Then the position and pose matrix of the end of the robot is:

$$
{ }_{4}^{0} T={ }_{1}^{0} T_{2}^{1} T_{3}^{2} T_{4}^{3} T=\left[\begin{array}{cccc}
n_{x} & o_{x} & a_{x} & p_{x} \\
n_{y} & o_{y} & a_{y} & p_{y} \\
n_{z} & o_{z} & a_{z} & p_{z} \\
0 & 0 & 0 & 1
\end{array}\right]
$$

After calculation,

$$
\begin{gathered}
n_{x}=c 4(c 1 c 2 c 3-c 1 s 2 s 3)-s 4(c 1 c 2 s 3+c 1 s 2 c 3) \\
n_{y}=c 4(s 1 c 2 c 3-s 1 s 2 s 3)-s 4(s 1 c 2 s 3+s 1 s 2 c 3) \\
n_{z}=c 4(c 2 s 3+s 2 c 3)+s 4(c 2 c 3-s 2 s 3) \\
o_{x}=-c 4(c 1 c 2 s 3+c 1 s 2 c 3)-s 4(c 1 c 2 c 3-c 1 s 2 s 3) \\
o_{y}=-c 4(s 1 c 2 s 3+s 1 s 2 c 3)-s 4(s 1 c 2 c 3-s 1 s 2 s 3) \\
o_{z}=c 4(c 2 c 3-s 2 s 3)-s 4(c 2 s 3+s 2 c 3) \\
a_{x}=s 1 \\
a_{y}=-c 1 \\
a_{z}=0 \\
p_{x}=260 s 1+410 c 1 c 2+390 c 1 c 2 c 3-390 c 1 s 2 s 3 \\
p_{y}=410 s 1 c 2-260 c 1+390 s 1 c 2 c 3-390 s 1 s 2 s 3 \\
p_{z}=410 s 2+390 c 2 s 3+390 s 2 c 3+250
\end{gathered}
$$

In these equations, $c 1=\cos \theta_{1}, s 1=\sin \theta_{1}, c 2=$ $\cos \theta_{2}, \quad s 2=\sin \theta_{2}, \quad c 3=\cos \theta_{3}, \quad s 3=\sin \theta_{3}, \quad c 4=$ $\cos \theta_{4}, s 4=\sin \theta_{4}$.

\subsubsection{Velocity analysis}

It is necessary to know the relationship between the plantar velocity and the motion speed of each joint in the control process of the rehabilitation robot. The Jacobian matrix is the transformation matrix of the robot's end effector speed and joint velocity [3]. It can be regarded as the speed transmission ratio from the joint space to the operating space. The kinematic equation of the mechanical leg is $x=x(q)$. The derivative of time $t$ on both sides of the equation is calculated. equation $\dot{\mathrm{x}}=$
$\mathrm{J}(\mathrm{q}) \dot{\mathrm{q}}$ is derived. $\dot{\mathrm{x}}$ represents the velocity of the end foot $\dot{q}$ represents joint velocity. $J(q)$ represents the Jacobian matrix, element in Row i column $\mathrm{j}$ is $J_{i j}(q)=\frac{\partial x_{i}(q)}{\partial q_{j}}$. The base coordinate system of waist does not coincide with the coordinate system of thigh connecting rod. So there is a joint between the waist and hip joint. It is just not moving, which is equivalent to a lock of the joint. In this paper, $J(q)$ is a $6^{*} 4$ Jacobian matrix.

$$
\left[\begin{array}{l}
v \\
\omega
\end{array}\right]=J(q)\left[\begin{array}{l}
\dot{q}_{1} \\
\dot{q}_{2} \\
\dot{q}_{3} \\
\dot{q}_{4}
\end{array}\right]=\left[\begin{array}{llll}
J_{l 1} & J_{l 2} & J_{l 3} & J_{l 4} \\
J_{a 1} & J_{a 2} & J_{a 3} & J_{a 4}
\end{array}\right]\left[\begin{array}{c}
\dot{q}_{1} \\
\dot{q}_{2} \\
\dot{q}_{3} \\
\dot{q}_{4}
\end{array}\right]
$$

There is the following formula for the rotational joint

$$
{ }^{T} J_{l i}=\left[\begin{array}{l}
(p \times n)_{z} \\
(p \times o)_{z} \\
(p \times a)_{z}
\end{array}\right] \quad{ }^{T} J_{a i}\left[\begin{array}{c}
n_{z} \\
o_{z} \\
a_{z}
\end{array}\right]
$$

In the formula, $\vec{n}, \vec{o}, \vec{a}$ and $\vec{p}$ are the four column vectors of the matrix ${ }_{4}^{i} T$.According to the homogeneous transformation matrix between adjacent connecting rods, ${ }_{4}^{3} T,{ }_{4}^{2} T,{ }_{4}^{1} T,{ }_{4}^{0} T$ can be obtained. The above homogeneous transformation matrix elements are brought into the form, so that the following results can be obtained.

$$
\begin{aligned}
& J_{4}=\left[\begin{array}{llllll}
0 & 0 & 0 & 0 & 0 & 1
\end{array}\right]^{T} \\
& J_{3}=\left[\begin{array}{llllll}
0 & 0 & 0 & 0 & 0 & 1
\end{array}\right]^{T} \\
& J_{2}=\left[\begin{array}{llllll}
A * B & A * C & 0 & B & C & 0
\end{array}\right]^{-1} \\
& J_{1}=\left[\begin{array}{lllllll}
(A+250) * B & (A+250) * C & 0 & B & C & 0
\end{array}\right]^{-1}
\end{aligned}
$$

In these equations:

$\mathrm{A}=410 \mathrm{~s} 2+390 \mathrm{c} 2 \mathrm{~s} 3+390 \mathrm{c} 3 \mathrm{~s} 2$

$\mathrm{B}=\mathrm{cB} 4(\mathrm{c} 2 \mathrm{c} 3-\mathrm{s} 2 \mathrm{~s} 3)-\mathrm{s} 4(\mathrm{c} 2 \mathrm{~s} 3+\mathrm{c} 3 \mathrm{~s} 2)$

$\mathrm{C}=\mathrm{c} 4(\mathrm{c} 2 \mathrm{c} 3-\mathrm{s} 2 \mathrm{~s} 3)-\mathrm{s} 4(\mathrm{c} 2 \mathrm{~s} 3+\mathrm{c} 3 \mathrm{~s} 2)$

Thus Jacobian matrix $J=\left[\begin{array}{llll}J_{1} & J_{2} & J_{3} & J_{4}\end{array}\right]$ is obtained. The velocity of the end effector can be obtained by calculating the joint velocity by Jacobian matrix. Conversely, the speed of each joint can be solved by the velocity of the end effector. The calculation formula is $\dot{q}=J_{a}^{-1}(q) v$. The acceleration value of the joint and end effector can be obtained by re derivation. The calculation formula is $\ddot{q}=\left[\begin{array}{c}\dot{v} \\ \dot{\omega}\end{array}\right]=J^{-1}(\ddot{x}-\dot{J} \dot{q})$.

\subsection{Dynamic modeling and calculation}

Dynamics of exoskeleton mechanical legs is discussed in order to further discuss control problems [4]. The dynamic mathematical model of robot operation is mainly analysed by two theories. The first method is the dynamic equilibrium method of force. By using Newton Euler equation, the acceleration is obtained from kinematics and the internal forces are eliminated [5]. The second method is Lagrange's function balance method. This method only needs speed without internal force. In this paper, the second method is used to analyse the dynamics of exoskeleton mechanical legs.

The Lagrange function $\mathrm{L}$ is defined as the difference between the kinetic energy $\mathrm{K}$ and the potential energy $\mathrm{P}$ of the system. Its expression is as follows: 


$$
L=K-P
$$

The Lagrange equation in this paper is as follows:

$$
F_{i}=\frac{d}{d t} \frac{\partial L}{\partial \dot{q}_{l}}-\frac{\partial L}{\partial \dot{q}_{l}}, i=1,2,3,4
$$

Among them, $q_{i}$ is the coordinates of the kinetic energy and the potential energy. Symbol $\dot{q}_{l}$ represents the corresponding speed. Symbol $F_{i}$ represents the force or torque acting on the coordinates of $i$. The three link model of exoskeleton mechanical legs is shown in Figure 2.2. $m_{1}, m_{2}$ and $m_{3}$ represent the quality of the thigh bar, leg bar and foot in the picture. The points at the end of the connecting rod represent the quality. $d_{1}, d_{2}$ and $d_{3}$ are the length of the three connecting rods. $\theta_{1}, \theta_{2}$ and $\theta_{3}$ are generalized coordinates. Letter $g$ represents the acceleration of gravity.

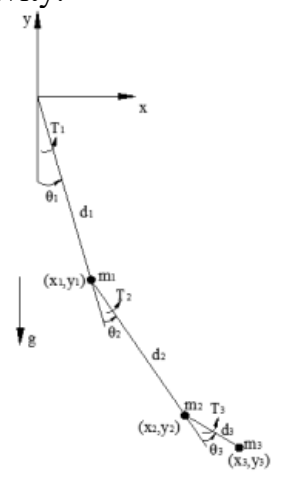

Figure 2.2. Three link model diagram of the mechanical leg

The kinetic energy $\mathrm{K}$ and the potential energy $\mathrm{P}$ of the thigh link can be expressed as:

$$
\begin{gathered}
K_{1}=\frac{1}{2} m_{1} d_{1}^{2} \dot{\theta}_{1}^{2} \\
P_{1}=-m_{1} g d_{1} \cos \theta_{1}
\end{gathered}
$$

The kinetic energy $\mathrm{K}$ and the potential energy $\mathrm{P}$ of the leg link can be expressed as:

$K_{2}=\frac{1}{2} m_{2} d_{1}^{2} \dot{\theta}_{1}^{2}+\frac{1}{2} m_{2} d_{2}^{2}\left(\dot{\theta}_{1}+\dot{\theta}_{2}\right)^{2}+$ $m_{2} d_{1} d_{2} \cos \theta_{2}\left(\dot{\theta}_{1}^{2}+\dot{\theta}_{1} \dot{\theta}_{2}\right)$

(2-18)

$P_{2}=-m_{2} g d_{1} \cos \theta_{1}-m_{2} g d_{2} \cos \left(\theta_{1}+\theta_{2}\right)$

The kinetic energy $\mathrm{K}$ and the potential energy $\mathrm{P}$ of the foot link can be expressed as:

$$
\begin{aligned}
& K_{3}=\frac{1}{2} m_{3} d_{1}^{2} \dot{\theta}_{1}^{2}+\frac{1}{2} m_{3} d_{2}^{2}\left(\dot{\theta_{1}}+\dot{\theta_{2}}\right)^{2}+\frac{1}{2} m_{3} d_{3}^{2}\left(\dot{\theta}_{1}+\right. \\
& \left.\dot{\theta_{2}}+\dot{\theta}_{3}\right)^{2}+m_{3} d_{1} d_{2} \dot{\theta}_{1}\left(\dot{\theta_{1}}+\dot{\theta_{2}}\right) \cos \theta_{2}+ \\
& m_{3} d_{1} d_{3} \dot{\theta}_{1}\left(\dot{\theta}_{1}+\dot{\theta}_{2}+\dot{\theta}_{3}\right) \cos \left(\theta_{2}+\theta_{3}\right)+ \\
& m_{3} d_{2} d_{3}\left(\dot{\theta}_{1}+\dot{\theta}_{2}\right)\left(\dot{\theta_{1}}+\dot{\theta}_{2}+\dot{\theta}_{3}\right) \cos \theta_{3} \\
& \quad(2-20)
\end{aligned}
$$$$
P_{3}=-m_{3} g d_{1} \cos \theta_{1}-m_{3} g d_{2} \cos \left(\theta_{1}+\theta_{2}\right)-
$$$$
m_{3} g d_{3} \cos \left(\theta_{1}+\theta_{2}+\theta_{3}\right)
$$

In this way, the total kinetic energy and total potential energy of the three link mechanical leg system are:

$$
\begin{gathered}
K=K_{1}+K_{2}+K_{3}=\frac{1}{2}\left(m_{1}+m_{2}+m_{3}\right) d_{1}^{2} \dot{\theta}_{1}^{2}+ \\
\frac{1}{2}\left(m_{2}+m_{3}\right) d_{2}^{2}\left(\dot{\theta}_{1}+\dot{\theta}_{2}\right)^{2}+\frac{1}{2} m_{3} d_{3}^{2}\left(\dot{\theta}_{1}+\dot{\theta}_{2}+\dot{\theta}_{3}\right)^{2}+ \\
\left(m_{2}+m_{3}\right) d_{1} d_{2} \dot{\theta}_{1}\left(\dot{\theta}_{1}+\dot{\theta_{2}}\right) \cos \theta_{2}+m_{3} d_{1} d_{3} \dot{\theta}_{1}\left(\dot{\theta_{1}}+\right. \\
\left.\dot{\theta_{2}}+\dot{\theta_{3}}\right) \cos \left(\theta_{2}+\theta_{3}\right)+m_{3} d_{2} d_{3}\left(\dot{\theta}_{1}+\dot{\theta}_{2}\right)\left(\dot{\theta}_{1}+\dot{\theta}_{2}+\right. \\
\left.\dot{\theta_{3}}\right) \cos \theta_{3} \\
P=P_{1}+P_{2}+P_{3}=-\left(m_{1}+m_{2}+m_{3}\right) g d_{1} \cos \theta_{1}- \\
\left(m_{2}+m_{3}\right) g d_{2} \cos \left(\theta_{1}+\theta_{2}\right)-m_{3} g d_{3} \cos \left(\theta_{1}+\theta_{2}+\right. \\
\left.\theta_{3}\right)
\end{gathered}
$$

The Lagrange function $\mathrm{L}$ of the three link mechanical leg system is obtained according to formula (2-14).

$$
\begin{gathered}
L=K-P=\frac{1}{2}\left(m_{1}+m_{2}+m_{3}\right) d_{1}^{2} \dot{\theta}_{1}^{2}+\frac{1}{2}\left(m_{2}+\right. \\
\left.m_{3}\right) d_{2}^{2}\left(\dot{\theta_{1}}+\dot{\theta}_{2}\right)^{2}+\frac{1}{2} m_{3} d_{3}^{2}\left(\dot{\theta_{1}}+\dot{\theta_{2}}+\dot{\theta_{3}}\right)^{2}+ \\
\left(m_{2}+m_{3}\right) d_{1} d_{2} \dot{\theta_{1}}\left(\dot{\theta_{1}}+\dot{\theta_{2}}\right) \cos \theta_{2}+m_{3} d_{1} d_{3} \dot{\theta}_{1}\left(\dot{\theta_{1}}+\right.
\end{gathered}
$$
$\left.\dot{\theta}_{2}+\dot{\theta_{3}}\right) \cos \left(\theta_{2}+\theta_{3}\right)+m_{3} d_{2} d_{3}\left(\dot{\theta_{1}}+\dot{\theta_{2}}\right)\left(\dot{\theta}_{1}+\dot{\theta}_{2}+\right.$ $\left.\dot{\theta_{3}}\right) \cos \theta_{3}+\left(m_{1}+m_{2}+m_{3}\right) g d_{1} \cos \theta_{1}+\left(m_{2}+\right.$ $\left.m_{3}\right) g d_{2} \cos \left(\theta_{1}+\theta_{2}\right)+m_{3} g d_{3} \cos \left(\theta_{1}+\theta_{2}+\theta_{3}\right)$ 24)

Take the corresponding derivative and partial derivative into the formula (2-15). Then the kinetic equations of hip joint, knee joint and ankle joint that be expressed by $\mathrm{T}_{1}, \mathrm{~T}_{2}$ and $\mathrm{T}_{3}$ can be obtained.

$$
\begin{aligned}
& T_{1}=\frac{d}{d t} \frac{\partial L}{\partial \dot{\theta_{1}}}-\frac{\partial L}{\partial \theta_{1}}=\left[\left(m_{1}+m_{2}+m_{3}\right) d_{1}^{2}+\left(m_{2}+\right.\right. \\
& \left.m_{3}\right) d_{2}^{2}+m_{3} d_{3}^{2}+2\left(m_{2}+m_{3}\right) d_{1} d_{2} \cos \theta_{2}+ \\
& \left.2 m_{3} d_{1} d_{3} \cos \left(\theta_{2}+\theta_{3}\right)+2 m_{3} d_{2} d_{3} \cos \theta_{3}\right] \ddot{\theta}_{1}+ \\
& {\left[\left(m_{2}+m_{3}\right) d_{2}^{2}+m_{3} d_{3}^{2}+\left(m_{2}+m_{3}\right) d_{1} d_{2} \cos \theta_{2}+\right.} \\
& \left.m_{3} d_{1} d_{3} \cos \left(\theta_{2}+\theta_{3}\right)+2 m_{3} d_{2} d_{3} \cos \theta_{3}\right] \ddot{\theta}_{2}+ \\
& {\left[m_{3} d_{3}^{2}+m_{3} d_{1} d_{3} \cos \left(\theta_{2}+\theta_{3}\right)+m_{3} d_{2} d_{3} \cos \theta_{3}\right] \ddot{\theta}_{3}-} \\
& 2\left[\left(m_{2}+m_{3}\right) d_{1} d_{2} \sin \theta_{2}+m_{3} d_{1} d_{3} \sin \left(\theta_{2}+\right.\right. \\
& \left.\left.\theta_{3}\right)\right] \dot{\theta}_{1} \dot{\theta}_{2}-2\left[m_{3} d_{1} d_{3} \sin \left(\theta_{2}+\theta_{3}\right)+\right. \\
& \left.m_{3} d_{2} d_{3} \sin \theta_{3}\right] \dot{\theta}_{2} \dot{\theta}_{3}-2\left[m_{3} d_{1} d_{3} \sin \left(\theta_{2}+\theta_{3}\right)+\right. \\
& \left.m_{3} d_{2} d_{3} \sin \theta_{3}\right] \dot{\theta_{1}} \dot{\theta_{3}}-\left[\left(m_{2}+m_{3}\right) d_{1} d_{2} \sin \theta_{2}+\right. \\
& \left.m_{3} d_{1} d_{3} \sin \left(\theta_{2}+\theta_{3}\right)\right] \dot{\theta}_{2}^{2}-\left[m_{3} d_{1} d_{3} \sin \left(\theta_{2}+\theta_{3}\right)+\right. \\
& \left.m_{3} d_{2} d_{3} \sin \theta_{3}\right] \dot{\theta}_{3}^{2}+\left(m_{1}+m_{2}+m_{3}\right) g d_{1} \sin \theta_{1}+ \\
& \left(m_{2}+m_{3}\right) g d_{2} \sin \left(\theta_{1}+\theta_{2}\right)+m_{3} g d_{3} \sin \left(\theta_{1}+\theta_{2}+\right. \\
& \left.\theta_{3}\right) \\
& T_{2}=\frac{d}{d t} \frac{\partial L}{\partial \dot{\theta}_{2}}-\frac{\partial L}{\partial \theta_{2}}=\left[\left(m_{2}+m_{3}\right) d_{2}^{2}+m_{3} d_{3}^{2}+\left(m_{2}+\right.\right. \\
& \left.m_{3}\right) d_{1} d_{2} \cos \theta_{2}+m_{3} d_{1} d_{3} \cos \left(\theta_{2}+\theta_{3}\right)+ \\
& \left.2 m_{3} d_{2} d_{3} \cos \theta_{3}\right] \ddot{\theta}_{1}+\left[\left(m_{2}+m_{3}\right) d_{2}^{2}+m_{3} d_{3}^{2}+\right. \\
& \left.2 m_{3} d_{2} d_{3} \cos \theta_{3}\right] \ddot{\theta}_{2}+\left(m_{3} d_{3}^{2}+m_{3} d_{2} d_{3} \cos \theta_{3}\right) \ddot{\theta}_{3}- \\
& 2 m_{3} d_{2} d_{3} \sin \theta_{3} \dot{\theta}_{1} \dot{\theta}_{3}-2 m_{3} d_{2} d_{3} \sin \theta_{3} \dot{\theta}_{2} \dot{\theta}_{3}+\left[\left(m_{2}+\right.\right. \\
& \left.\left.m_{3}\right) d_{1} d_{2} \sin \theta_{2}+m_{3} d_{1} d_{3} \sin \left(\theta_{2}+\theta_{3}\right)\right] \dot{\theta}_{1}^{2}- \\
& m_{3} d_{2} d_{3} \sin \dot{\theta}_{3} \dot{\theta}_{3}^{2}+\left(m_{2}+m_{3}\right) g d_{2} \sin \left(\theta_{1}+\theta_{2}\right)+ \\
& m_{3} g d_{3} \sin \left(\theta_{1}+\theta_{2}+\theta_{3}\right) \\
& (2-26)
\end{aligned}
$$

$T_{3}=\frac{d}{d t} \frac{\partial L}{\partial \dot{\theta}_{3}}-\frac{\partial L}{\partial \theta_{3}}=\left[m_{3} d_{3}^{2}+m_{3} d_{1} d_{3} \cos \left(\theta_{2}+\theta_{3}\right)+\right.$ $\left.m_{3} d_{2} d_{3} \cos \theta_{3}\right] \ddot{\theta_{1}}+\left(m_{3} d_{3}^{2}+m_{3} d_{2} d_{3} \cos \theta_{3}\right) \ddot{\theta_{2}}+$ $m_{3} d_{3}^{2} \ddot{\theta}_{3}+2 m_{3} d_{2} d_{3} \sin \theta_{3} \dot{\theta_{1}} \dot{\theta}_{2}+\left[m_{3} d_{1} d_{3} \sin \left(\theta_{2}+\right.\right.$ $\left.\left.\theta_{3}\right)+m_{3} d_{2} d_{3} \sin \theta_{3}\right] \dot{\theta}_{1}^{2}+m_{3} d_{2} d_{3} \sin \theta_{3} \dot{\theta}_{2}^{2}+$ $m_{3} g d_{3} \sin \left(\theta_{1}+\theta_{2}+\theta_{3}\right)$

\section{Structure design}

The human body is mainly in sagittal plane when walking normally. Flexion and extension of the hip joint, knee joint and ankle joint are the main driving force of the movement. In this paper, an exoskeleton rehabilitation robot with three degree of freedom is designed to meet the needs of normal walking of human body. The flexion or extension of the hip joint, the knee joint and the ankle joint are the active rotational degrees of freedom. The disc motor is selected to drive the three joints. In addition, the torque is increased by 
the harmonic reducer. The lower limb rehabilitation robot designed in this paper has five parts including the system framework, the weight reduction mechanism, the balance mechanism, the exoskeleton mechanical leg and a treadmill.

\subsection{D modeling}

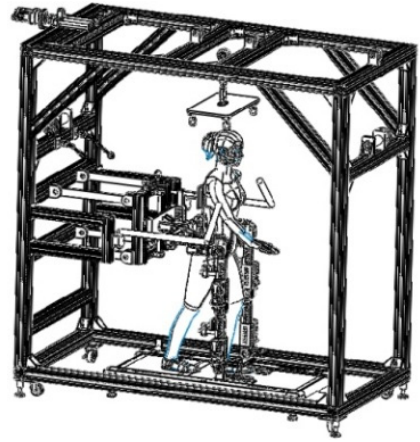

Figure 3.1. Model map of lower limb rehabilitation robot The system frame is constructed by $80 * 80$ aluminum alloy profiles. The assembly part of the profile is connected with the corners, which is used to strengthen the supporting force of the points connected to the profiles. The handrail is fixed on the frame with an emergency stop button on it, which can stop operation under sudden condition to ensure the patient's personal safety. The alloy hoofs support the whole frame.

The weight reduction mechanism can help patients reduce their own weight and avoid pressing their lower extremities. One end of the wire rope is attached to the $T$ nut connected with the screw rod, and the other end passes through the pulley block to wrap on the windlass installed on the front. The patient wore a vest and was suspended on a flat plate connected under a movable pulley, which was connected with a Tianmu NS-WL1 tension pressure sensor between the movable pulley and the flat plate. When walking on the treadmill, the central of gravity of the body has the offset of positive and negative $50 \mathrm{~mm}$ in the vertical direction [6]. So it is necessary to control the motor synchronously according to the position of the body's central of gravity. The motor drives the lead screw to rotate, so as to realize the movement of the $\mathrm{T}$ nut and maintain the tension of the wire rope connected by the $\mathrm{T}$ nut. The balance mechanism is mounted on the frame, and one end of the balancing mechanism is fixedly connected with the exoskeleton mechanical leg. It allows patients to move up and down and restrict left and right movements to ensure the body balance during walking. Suspension by wire rope can reduce the weight of the mechanism and avoid additional burden on the lower extremities.

The hip, knee and ankle joints of exoskeleton mechanical legs are driven by six disc motors and harmonic decelerators respectively. In addition, each joint is equipped with an angle sensor. According to the national standard GB/T10000-1988 "Chinese adult body size", the mechanical leg adjustable length range is designed. The adjustable range of the thigh rod is $350 \sim 470 \mathrm{~mm}$. The adjustable range of the leg length is $330 \sim 450 \mathrm{~mm}$. The Shanghai Jiuzhi L10M-100KG pressure sensor is arranged in the middle of the double foot pedals of the mechanical legs. The plantar force and related time parameters of patients in exercise state were measured. This is beneficial for medical clinical diagnosis and evaluation of rehabilitation treatment results.

\subsection{Selection and calculation of driving mode}

The maximum output torque of the ankle joint is $15 \mathrm{Nm}$. Rehabilitation training requires that ankle dorsiflexion or plantar flexion should be completed in more than 6 seconds, Then the speed of the reducer output is required to be $0.29 \mathrm{rad} / \mathrm{s}$. Therefore, the basic power of the output is $0.29 * 15=4.36 \mathrm{w}$. According to the sample of Maxon motor products, EC45 disc $30 \mathrm{~W}$ brushless motor is selected. This type of motor has a rated output torque of $59 \mathrm{Nm}$ and a rated speed of 2860rpm. The harmonic reducers from Beijing Institute of Harmonics with the reduction ratio of 160 were selected. The model is XB31-63-160. The motor rotation speed is 443rpm . According to the motor characteristic curve, the output torque of the motor is $125 \mathrm{mNm}$ as soon as the motor reaches $443 \mathrm{rpm}$ speed. In the meantime, the output torque of the joint is $20 \mathrm{Nm}$ after harmonic reduction. The harmonic reducer can withstand the torque of $30 \mathrm{Nm}$, which completely meets the design requirements. Similarly, the Maxon motor EC90 disc 260W brushless motor is selected for the knee joint. In addition, a harmonic reducer with a reduction ratio of 50 is selected. The model is XB3-1-100-50. The Maxon motor EC90 disc $260 \mathrm{~W}$ brushless motor is selected for the hip joint. In addition, a harmonic reducer with a reduction ratio of 70 is selected. The model is XB3-1-100-70.

\section{Conclusion}

To achieve rehabilitation, we performed dynamic analysis and kinematic analysis of the lower limb rehabilitation robot. According to the analysis, the lower limb rehabilitation robot designed in this paper has five parts, including the system framework, the weight reduction mechanism, the balance mechanism, the exoskeleton mechanical leg and a treadmill. The Maxon EC45 disc 30W brushless motor is selected for the ankle, both keen and hip joint are equipped with Maxon motor EC90 disc 260W brushless motor. In order to get the real time information of human body, Shanghai Jiuzhi L10M-100KG pressure sensor is arranged in the middle of the double foot pedals of the mechanical legs.

\section{References}

1. Wang Jun. Design and Simulation of lower limb reinforcement mechanism of exoskeleton robot [D]. Zhejiang University, 2016.

2. Li Qing, Mao Jian, Zhu Zina,Zhou Yufeng Cooperative motion model of two manipulators and workspace analysis[J].Manufacturing Automation, 2017,39(11): 44-48+55. 
3. Liu Hongtao. Design and experimental study of lower limb rehabilitation robot for paraplegia [D]. Yanshan University, 2010.

4. Yin Junmao. Analysis and design of wearable lower limb exoskeleton [D]. Beijing University of Technology, 2010.

5. Cai Zixing, Xie Bin. Robotics $[\mathrm{M}]$. Beijing: Tsinghua University press, 2000

6. Chen. Research on experimental prototype of weight loss walking training robot [D]. Tsinghua University, 2010. 